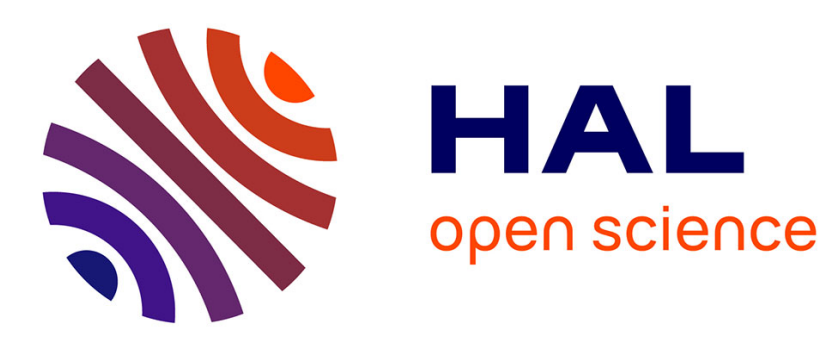

\title{
Simulation et analyse d'une structure non-linéaire à symétrie cyclique
}

Aurélien Grolet, Fabrice Thouverez, Pierrick Jean

\section{To cite this version:}

Aurélien Grolet, Fabrice Thouverez, Pierrick Jean. Simulation et analyse d'une structure non-linéaire à symétrie cyclique. Mécanique et Industries, 2010, 11 (6), pp.453-463. 10.1051/meca/2010047 . hal-02424289

\section{HAL Id: hal-02424289 \\ https://hal.science/hal-02424289}

Submitted on 27 Dec 2019

HAL is a multi-disciplinary open access archive for the deposit and dissemination of scientific research documents, whether they are published or not. The documents may come from teaching and research institutions in France or abroad, or from public or private research centers.
L'archive ouverte pluridisciplinaire HAL, est destinée au dépôt et à la diffusion de documents scientifiques de niveau recherche, publiés ou non, émanant des établissements d'enseignement et de recherche français ou étrangers, des laboratoires publics ou privés.

\section{(c)(1)}

Distributed under a Creative Commons Attribution| 4.0 International License 


\title{
Simulation et analyse d'une structure non-linéaire à symétrie cyclique
}

\author{
Aurélien Grolet ${ }^{1, a}$, Fabrice Thouverez ${ }^{1}$ et Pierrick Jean ${ }^{2}$ \\ 1 École Centrale de Lyon, Laboratoire de Tribologie et Dynamique des Systèmes, 36 avenue Guy de Collongue, \\ 69134 Écully Cedex, France \\ 2 Snecma - Safran group, 77550 Moissy-Cramayel, France
}

\begin{abstract}
Résumé - Cet article est dédié à l'étude des vibrations libres et forcées d'une structure à symétrie cyclique, soumise à des non-linéarités géométriques, par la méthode de la balance harmonique (HBM). Dans le but d'étudier l'influence des non-linéarités un modèle simplifié a été développé. Après ajustement des paramètres du modèle, les équations du mouvement se présentent sous la forme d'équations différentielles du second ordre, linéairement couplées, où les non-linéarités se traduisent par des termes polynomiaux d'ordre deux et trois. Les solutions périodiques de ces équations sont recherchées grâce à la méthode de la balance harmonique couplée avec une procédure de continuation. Dans le cas libre, en plus des modes non-linéaires similaires et non similaires, on met en évidence des modes non-linéaires localisés. Dans le cas forcé, plusieurs types d'excitations sont considérées (excitation sur le premier mode propre linéaire et excitation detunée) et on étudie particulièrement l'influence du niveau d'excitation sur la structure des réponses dynamiques. Pour une excitation suffisamment perturbée, on montre que plusieurs solutions peuvent coexister, certaines d'entre elles étant représentées par des courbes fermées dans le plan fréquenceamplitude.
\end{abstract}

Mots clés : Non-linéarité géométrique / mode non-linéaire / bifurcation / localisation

\begin{abstract}
Simulation and analysis of a nonlinear structure with cyclic symmetry. This paper is intented to study both free and forced vibration of a nonlinear structure with cyclic symmetry, under geometric nonlinearity, through use of the harmonic balance method (HBM). In order to study the influence of nonlinearity due to the large deflection of blades, a simplified model has been developed. After adjusting the model parameters, this approach leads to a system of linearly-coupled, second-order nonlinear differential equations, in which nonlinearity appears via quadratic and cubic terms. Periodic solutions, are sought by applying HBM coupled with an arc length continuation method. In the free case, in addition to featuring similar and nonsimilar nonlinear modes, the unforced system is shown to contain localized nonlinear modes. In the forced case, several cases of excitation have been analyzed (low-engine-order excitation and detuned excitation) and we study the influence of the excitation level on the structure of dynamical response. For a sufficiently-detuned excitation, we show that several solutions can coexist, some of them being represented by closed curves in the frequency-amplitude domain.
\end{abstract}

Key words: Geometric non-linearity / nonlinear normal mode / bifurcation / localization

\section{Introduction}

Cet article a pour objet l'étude des vibrations nonlinéaires libres et forcées d'une structure à symétrie cyclique composée de sous-structures identiques, soumises à

\footnotetext{
a Auteur pour correspondance :

aurelien.grolet@ecl2009.ec-lyon.fr
}

de grandes déformations. Cette classe de système apparaît en particulier dans la modélisation des roues aubagées [1]. La mise en équation de telles structures, conduit à un système d'équations différentielles couplées, d'ordre 2, avec des termes non-linéaires polynomiaux.

Dans le cas linéaire, en raison de la symétrie parfaite du problème, l'étude de ces équations fait apparaître en 


\section{Nomenclature}

\begin{tabular}{|ll|}
\hline $\boldsymbol{A}_{k}, \boldsymbol{B}_{k}$ & Coefficients de la HBM \\
$\boldsymbol{F}(t)$ & Forces externes dans le domaine temporel. \\
$\boldsymbol{G}(\boldsymbol{Y}, \omega)$ & Système d'équation algébrique issu de la HBM \\
$\mathbf{J}_{Y}$ & Matrice jacobienne de $\boldsymbol{G}$ par rapport à $\boldsymbol{Y}$ \\
$\mathbf{M}, \mathbf{K}$ & Matrices de masse et de raideur \\
$\boldsymbol{X}(t)$ & Vecteur des d.d.l. dans le domaine temporel \\
$\boldsymbol{Y}$ & Vecteur des coefficients de la HBM \\
$n_{p}$ & Nombre de secteurs \\
$w_{j}$ & Déplacement transverse du secteur $j(m)$ \\
$x_{j}$ & Coordonnée de Ritz pour le secteur $j($ d.d.l. $)(m)$ \\
$\delta$ & Coefficient d'amortissement \\
$\Phi_{i}^{\text {c }}, \Phi_{i}^{\text {s }}$ & Forme propre linéaire \\
$\Phi$ & Fonction d'interpolation de Ritz \\
$\omega, \Omega$ & Fréquence libre, fréquence d'excitation (rad.s $\left.{ }^{-1}\right)$ \\
\hline
\end{tabular}

majorité des fréquences propres doubles correspondant à des formes propres distinctes associées à des modes de vibration à diamètre [2].

Dans le cas non-linéaire, l'étude des vibrations libres repose sur la définition des modes non-linéaires (MNL) [3,4]. Contrairement au système linéaire, le nombre de modes non-linéaires peut être supérieur au nombre de degrés de liberté du système, les modes supplémentaires prenant naissance au travers de bifurcations. Dans [1,5], Peeters utilise une méthode de tir pour calculer les modes normaux non-linéaires d'un système à symétrie cyclique et met en évidence des modes similaires et non similaires et montre l'existence d'interactions modales.

Dans le cas libre ou forcé, si le couplage entre les sousstructures est suffisamment faible ou si la non-linéarité est suffisamment forte, Vakakis [6] a montré que des phénomènes de localisation sont possibles pour des structures cycliques parfaitement symétriques. Lorsque le ratio entre coefficient de couplage et coefficient de nonlinéarité augmente, des bifurcations apparaissent et les phénomènes de localisation se dissipent.

Dans le cadre de cet article, nous nous proposons d'étudier la réponse libre et forcée d'un modèle simplifié de roue aubagée en s'appuyant sur une approche de type balance harmonique couplée à une procédure de continuation. Une attention particulière sera portée sur l'influence du niveau de chargement sur la réponse forcée.

\section{Mise en équation}

\subsection{Modèle simplifié de roue aubagée}

Dans un premier temps, on considère une structure de type roue aubagée dont les $n_{p}=6$ secteurs sont modélisés par des plaques minces rectangulaires identiques (Fig. 1). Cette structure nous permettra d'obtenir une forme générale des équations du mouvement pour des structures à symétrie cyclique.

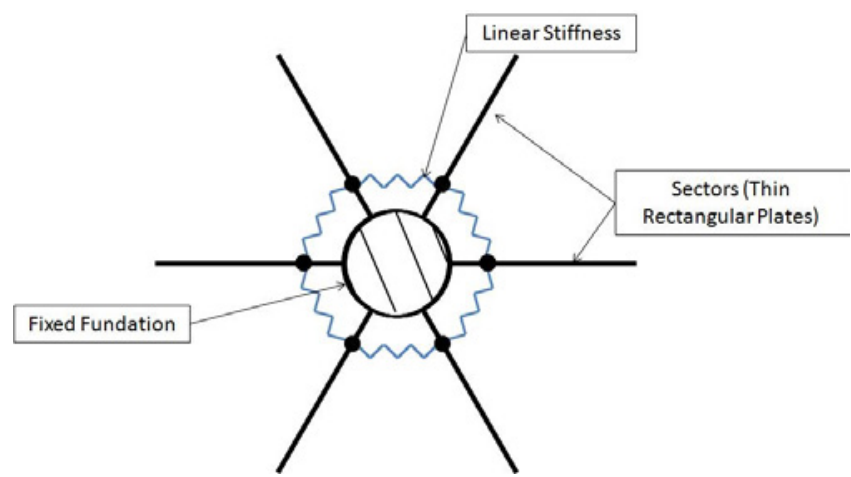

Fig. 1. Modèle de roue aubagée utilisé pour la mise en équation.

Chaque secteur est soumis à des non-linéarités géométriques $[7,8]$. On suppose de plus l'existence d'un couplage entre chaque secteur réalisé par une raideur linéaire qui agit entre deux secteurs consécutifs. En utilisant les hypothèses de Love Kirchhoff, on ne retient comme inconnu que le déplacement transverse de chaque secteur $j$ noté $w_{j}\left(1 \leq j \leq n_{p}\right)$. L'expression de la nonlinéarité est donnée par la relation de Von Karman en supposant que le matériau suit une loi de Hooke standard [7]. La mise en équation du système est réalisée grâce aux équations de Lagrange. Après avoir calculé une expression du lagrangien en fonction des déplacements transverses de chaque secteur, on discrétise le système en s'appuyant sur une approche de Ritz [9]. On ne retient qu'une seule fonction pour interpoler les déplacements de telle manière que l'on ait $w_{j}=x_{j} \Phi(x)$ où $\Phi(x)=\left(\frac{x}{L_{x}}\right)^{2}$ est la fonction de Ritz choisie, avec $L_{x}$ la longueur de la plaque. Dans ce cas la variable $x_{j}$ correspond à la flèche en bout de pale pour le secteur $j$ et les équations du mouvement dans le domaine temporel s'écrivent alors :

$$
\ddot{\boldsymbol{X}}+\mathbf{K} \boldsymbol{X}+b \boldsymbol{X}^{3}=\boldsymbol{F}(t)
$$

avec $\boldsymbol{X}=\left(x_{j}\right)_{1 \leq j \leq 6}$, la convention d'écriture $\boldsymbol{X}^{3}=$ $\left(x_{j}^{3}\right)_{1 \leq j \leq 6}$, et $\mathbf{K}$ une matrice circulante, caractéristique des systèmes à symétrie cyclique, définie par :

$$
\mathbf{K}=\left(\begin{array}{cccccc}
a+2 c & -c & 0 & 0 & 0 & -c \\
-c & a+2 c & -c & 0 & 0 & 0 \\
0 & -c & a+2 c & -c & 0 & 0 \\
0 & 0 & -c & a+2 c & -c & 0 \\
0 & 0 & 0 & -c & a+2 c & -c \\
-c & 0 & 0 & 0 & -c & a+2 c
\end{array}\right)
$$

où les coefficients $a, c$ et $b$, ainsi que le vecteur $\boldsymbol{F}$ sont définis en annexe.

\subsection{Ajustement des paramètres de l'équation}

Une étude des paramètres de l'équation (1) nous montre que le système obtenu est fortement non-linéaire. 


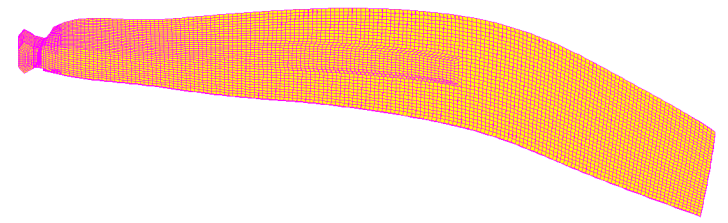

Fig. 2. Modèle éléments-finis d'un secteur.

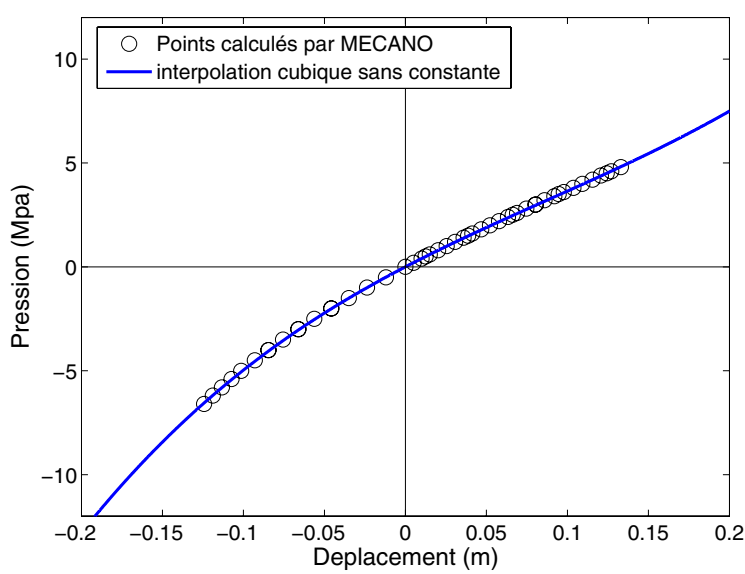

Fig. 3. Point de calcul et interpolation de la relation entre pression et déplacement transverse.

Cette forte non-linéarité est due à la fonction de Ritz $\Phi$ choisie pour interpoler les déplacements. De plus, ces équations ne sont valables que dans le cas où les secteurs sont parfaitement symétriques (pas de terme quadratique).

On souhaite ajuster les paramètres de l'équation (1) en se basant sur une structure réelle dont on dispose d'un modèle éléments finis (Fig. 2). La méthode d'ajustement consiste à rechercher une relation simplifiée entre force et déplacement qui conduira à une équation approchée pour le premier mode de flexion d'un secteur. Les autres secteurs seront ensuite pris en compte au travers d'un coefficient de couplage pour finalement obtenir les équations du mouvement d'une structure à symétrie cyclique composée de secteurs réels (non symétriques) vibrant selon leur premier mode de flexion. Étant donné que la structure réelle n'est pas parfaitement symétrique, on s'attend à obtenir des termes quadratiques dans l'équation du mouvement.

Pour déterminer la relation simplifiée entre force et déplacement pour la pale réelle, on applique un champ de pression statique uniforme sur toute la structure selon la direction transverse, et on relève le déplacement en bout de pale selon cette même direction. On obtient un nuage de points, qui est ensuite interpolé par un polynôme de degré 3 (Fig. 3). La relation obtenue entre la pression $P$ (en $\mathrm{MPa}$ ) et le déplacement en bout de pale $x$ (en m) est de la forme :

$$
P(x)=a_{3} x^{3}+a_{2} x^{2}+a_{1} x
$$

avec $a_{3}=254,90, a_{2}=-66,30, a_{1}=40,54$.
En ne considérant qu'une seule pale et en prenant en compte la relation de l'équation (3), on suppose que l'équation du mouvement pour le premier mode de flexion est de la forme suivante :

$$
\ddot{x}+\alpha x+\gamma x^{2}+\beta x^{3}=f(t) \Leftrightarrow \ddot{x}+F(x)=f(t)
$$

avec $x$ le déplacement transverse en bout de pale, $f(t)$ la force d'excitation, $\alpha=\omega_{0}^{2}$ et $\omega_{0}$ la fréquence propre du premier mode de flexion calculé par le module dynam de Samcef.

On transforme la relation entre pression et déplacement de l'équation (3) en une relation entre force et déplacement en utilisant la valeur de la fréquence du premier mode de flexion. On suppose que la force liée au déplacement est de la forme $F(x)=\chi P(x)$ avec $\chi$ une constante. En identifiant les termes de l'équation (4) on obtient :

$$
\chi=\frac{\alpha}{a_{1}}, \gamma=\chi a_{2}, \beta=\chi a_{3}
$$

Les équations du mouvement pour chaque secteur $j(j=$ 1 à 6) sont alors données par :

$$
\ddot{x}_{j}+(\alpha+2 c) x_{j}-c x_{j-1}-c x_{j+1}+\gamma x_{j}^{2}+\beta x_{j}^{3}=f_{j}(t)
$$

où $c$ représente la valeur du coefficient de couplage et $f_{j}$ la force d'excitation sur le secteur $j$.

En remplaçant $a$ par $\alpha$ dans la définition de la matrice K (Éq. (2)), l'équation (6) peut se réécrire sous forme matricielle :

$$
\ddot{\boldsymbol{X}}+\mathbf{K} \boldsymbol{X}+\gamma \boldsymbol{X}^{2}+\beta \boldsymbol{X}^{3}=\boldsymbol{F}(t)
$$

Les valeurs numériques des paramètres obtenues après l'opération d'ajustement sont données par :

$$
\begin{aligned}
& \alpha=\omega_{0}^{2}=14298 \mathrm{rad}^{2} \cdot \mathrm{s}^{-2} \quad \gamma=-23300 \mathrm{~m}^{-1} \cdot \mathrm{s}^{-2} \\
& \beta=89898 \mathrm{~m}^{-2} \cdot \mathrm{s}^{-2}
\end{aligned}
$$

Le coefficient de couplage est choisi arbitrairement à $c=$ $150 \mathrm{rad}^{2} . \mathrm{s}^{-2}$.

\section{Méthode de résolution : «harmonic balance method »}

La méthode de la balance harmonique est une technique très utilisée pour étudier les systèmes non-linéaires et ses exemples d'application sont nombreux. Cette méthode permet de traiter le cas de systèmes fortement non-linéaires avec par exemple du frottement [10,11], ou des non-linéarités géométriques $[12,13]$.

La méthode de la balance harmonique consiste à rechercher les solutions périodiques $\boldsymbol{X}$ de l'équation (7) sous forme de séries de Fourier tronquées :

$$
\boldsymbol{X}(t)=\boldsymbol{A}_{0}+\sum_{k=1}^{N_{h}} \boldsymbol{A}_{k} \cos (k \omega t)+\boldsymbol{B}_{k} \sin (k \omega t)
$$


En remplaçant l'approximation de l'équation (9) dans les équations du mouvement (7), et en identifiant les termes en sinus et cosinus, on obtient un système d'équations algébriques non-linéaires à $\left(2 N_{h}+1\right) n_{p}+1$ inconnues $\boldsymbol{A}_{k}$ $\boldsymbol{B}_{k}$ et $\omega$. Dans le cas où l'amplitude de la force d'excitation est périodique de pulsation $\Omega$, on prendra $\omega=\Omega$.

Le paramètre le plus important de cette méthode est le nombre d'harmoniques retenues $\left(N_{h}\right)$. Ce nombre n'est généralement pas connu a priori, ce qui nécessite des études de convergence pour assurer une bonne estimation de la solution. Cependant, dans un nombre important de cas, il s'avère que la solution converge vite en termes d'harmoniques ce qui permet d'avoir des systèmes de dimension raisonnable.

Le système obtenu après l'application de la HBM peut se réécrire sous la forme suivante :

$$
\boldsymbol{G}(\boldsymbol{Y}, \omega)=0
$$

où $\boldsymbol{Y}$ est un vecteur correspondant aux inconnues de la HBM et $\boldsymbol{G}$ une fonction non-linéaire définissant les équations algébriques.

L'équation (10) sera résolue par une technique de continuation par longueur d'arc [14]. La stabilité des solutions obtenues sera évaluée par une méthode de Floquet [14] et la détection des bifurcations s'opérera en observant le déterminant de la matrice jacobienne $\mathbf{J}_{\boldsymbol{Y}}=\frac{\partial \boldsymbol{G}}{\partial \boldsymbol{Y}}[14,15]$.

\section{Réponse libre du système à six degrés de liberté}

Notre premier objectif est d'estimer les solutions périodiques libres du système (7). L'approche naturelle est donc l'utilisation des modes non-linéaires (MNL) [3, $4,16]$.

Les fréquences propres et les formes propres du système linéaire sont données par (les fréquences sont données en rad.s $\left.{ }^{-1}\right)$ :

$$
\begin{aligned}
\omega_{0}=\sqrt{\alpha}=119,5742 \quad \Phi_{0} & =[1,1,1,1,1,1] \\
\omega_{1}=\sqrt{\alpha+c}=120,1998 \Phi_{1}^{c} & =[1,1,0,-1,-1,0] \\
\Phi_{1}^{s} & =\left[1, \frac{1}{2},-\frac{1}{2},-1,-\frac{1}{2}, \frac{1}{2}\right] \\
\omega_{2}=\sqrt{\alpha+3 c}=121,4413 \Phi_{2}^{c} & =[1,-1,0,1,-1,0] \\
\Phi_{2}^{s} & =\left[1,-\frac{1}{2},-\frac{1}{2}, 1-\frac{1}{2},-\frac{1}{2}\right] \\
\omega_{3}=\sqrt{\alpha+4 c}=122,0574 \Phi_{3} & =[1,-1,1,-1,1,-1]
\end{aligned}
$$

Les formes propres de l'équation (11) sont représentées sur la figure 4.

\subsection{Modes non-linéaires}

On recherche maintenant les modes non-linéaires du système (7). Les branches de solutions libres sont calculées

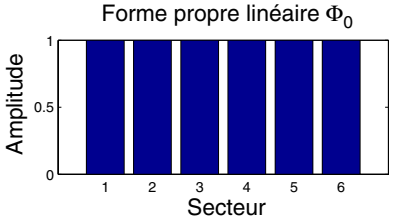

Forme propre linéaire $\Phi_{1}^{\mathrm{s}}$

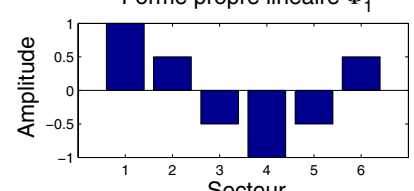

Forme propre linéaire $\Phi_{2}^{\mathrm{s}}$
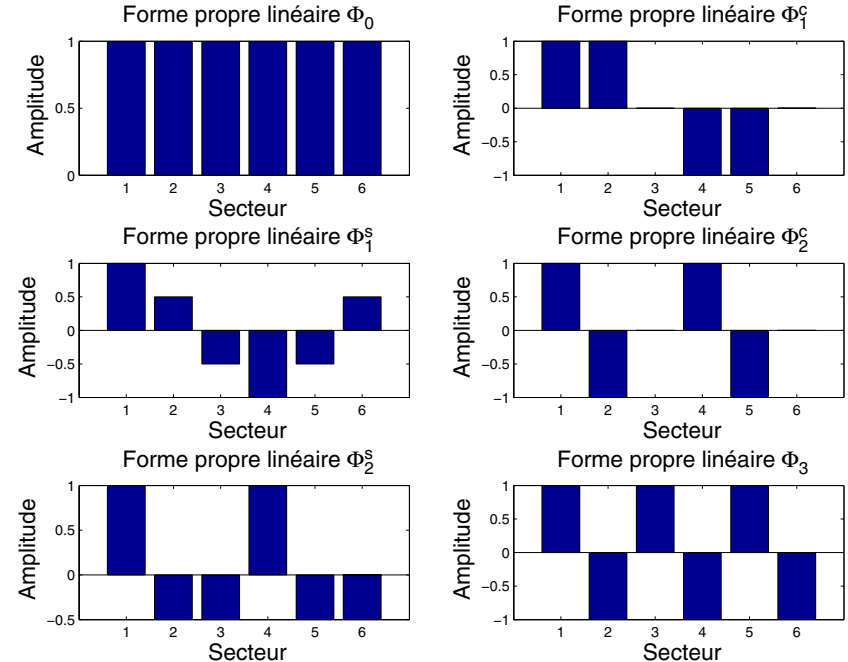

Forme propre linéaire $\Phi_{2}^{\complement}$

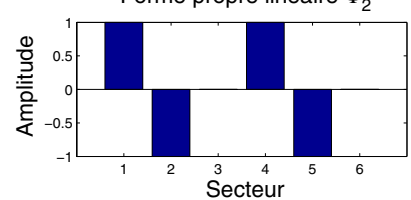

Forme propre linéaire $\Phi_{3}$

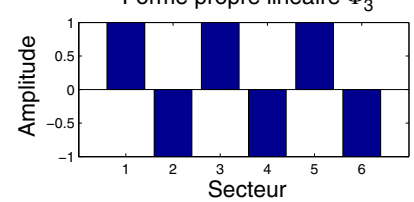

Fig. 4. Formes propres du système linéaire.

en choisissant comme point de départ un mode linéaire à basse amplitude de vibration. Les backbones curves, courbes présentant l'évolution de la fréquence de vibration en fonction de l'amplitude, seront représentées dans le plan énergie-fréquence [1]. L'énergie considérée ici est définie par :

$$
E(\boldsymbol{X})=\sqrt{\left\|\boldsymbol{A}_{0}\right\|^{2}+\sum_{k=1}^{N_{h}}\left(\left\|\boldsymbol{A}_{k}\right\|^{2}+\left\|\boldsymbol{B}_{k}\right\|^{2}\right)}
$$

où $\boldsymbol{A}_{k}$ et $\boldsymbol{B}_{k}$ sont les coefficients de la HBM définis à l'équation(9).

En choisissant une condition de phase dans laquelle toute les vitesses initiales sont nulles, les modes nonlinéaires du système (7) sont recherchés sous la forme suivante :

$$
\boldsymbol{X}(t)=\boldsymbol{A}_{0}+\boldsymbol{A}_{1} \cos \omega t+\boldsymbol{A}_{2} \cos 2 \omega t+\boldsymbol{A}_{3} \cos 3 \omega t
$$

\subsection{Modes non-linéaires « naturels »}

Les modes non-linéaires issus des modes linéaires sont représentés dans le plan énergie-fréquence sur les figures 5 et 6 . On observe bien qu'ils sont tangents aux modes propres linéaires et que la non-linéarité à un effet durcissant.

Les modes non-linéaires associés aux formes $\Phi_{i}^{c}(i=$ $0,1,2$, ou 3) sont des modes similaires, leur forme propre ne varie pas avec l'amplitude de vibration. En revanche les modes associés aux formes $\Phi_{1}^{s}$ et $\Phi_{2}^{s}$ ne sont pas similaires et le premier tend vers un mode de vibration où toutes les amplitudes sont les mêmes, alors que le second tend vers un mode de vibration qui localise le mouvement sur deux pales seulement (Fig. 6).

\subsection{Modes non-linéaires localisés}

Après étude du jacobien pour le mode non-linéaire à 3 diamètres, on met en évidence des bifurcations de 


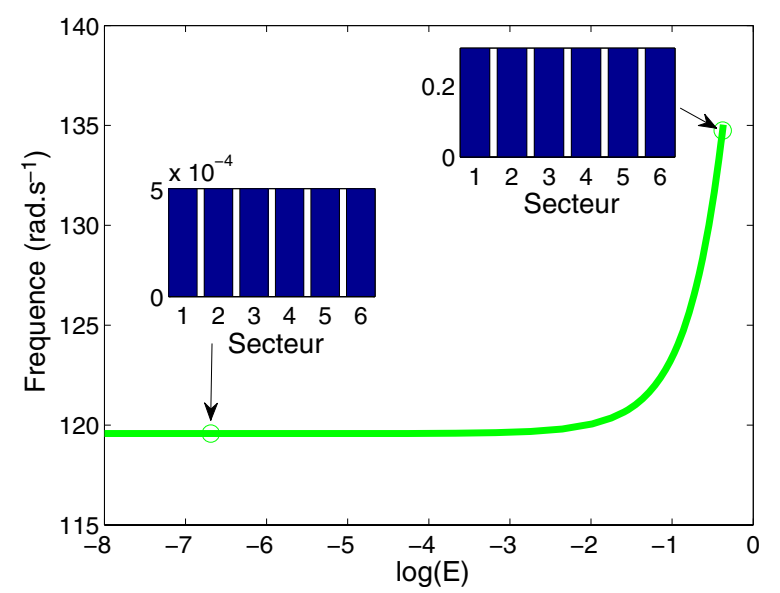

(a) MNL 0 diamètre

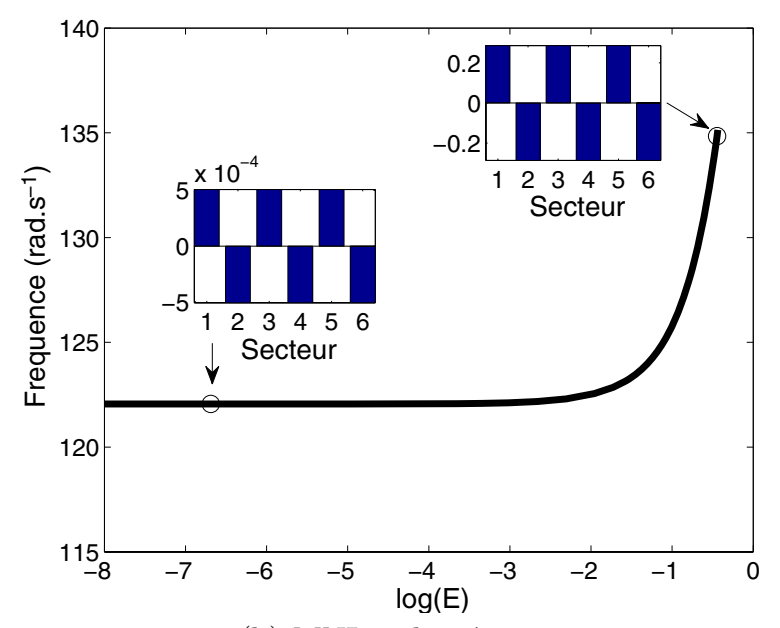

(b) MNL 3 diamètres

Fig. 5. Backbone curves et formes propres pour les modes à 0 et 3 diamètres.

type point d'embranchement. Les résultats sont donnés sur la figure 7 où la backbone curve du mode non-linéaire à 3 diamètres est représentée en trait continu et les bifurcations en traits pointillés. Les formes propres de ces différents modes de vibrations localisés sont représentées sur la figure 8 pour une pulsation d'environ $135 \mathrm{rad}^{-1}{ }^{-1}$.

On peut en conclure que les solutions localisées sur 1, 2, 3 ou 4 pales prennent naissance à partir du mode à 3 diamètres au travers de bifurcations de type point d'embranchement.

\section{Réponse forcée}

Les résultats de la partie précédente (solutions libres), ont permis de retrouver les différents modes de vibration présentés dans la littérature pour des structures à symétrie cyclique soumises à des non-linéarités [6]. Ces résultats ont montré une grande complexité dans le comportement vibratoire libre de telles structures. Bien

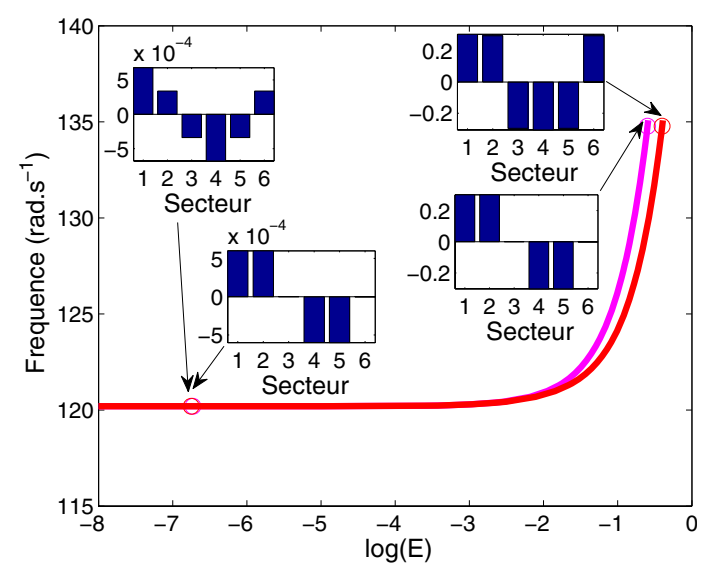

(a) MNL 1 diamètre

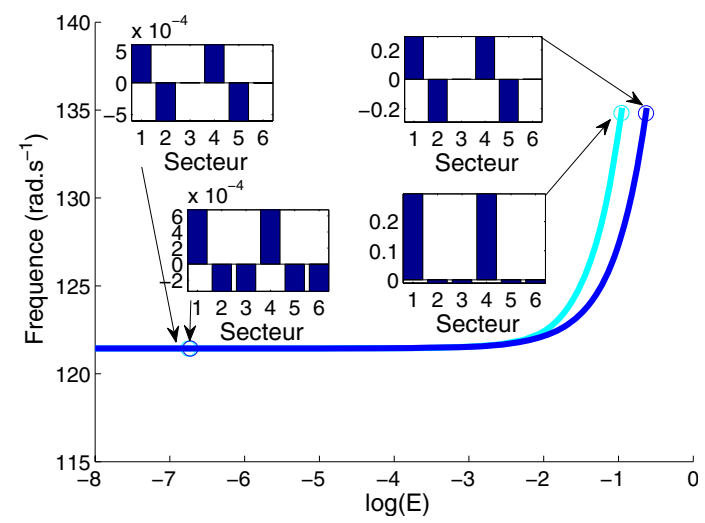

(b) MNL 2 diamètres

Fig. 6. Backbone curves et formes propres pour les modes à 1 et 2 diamètres.

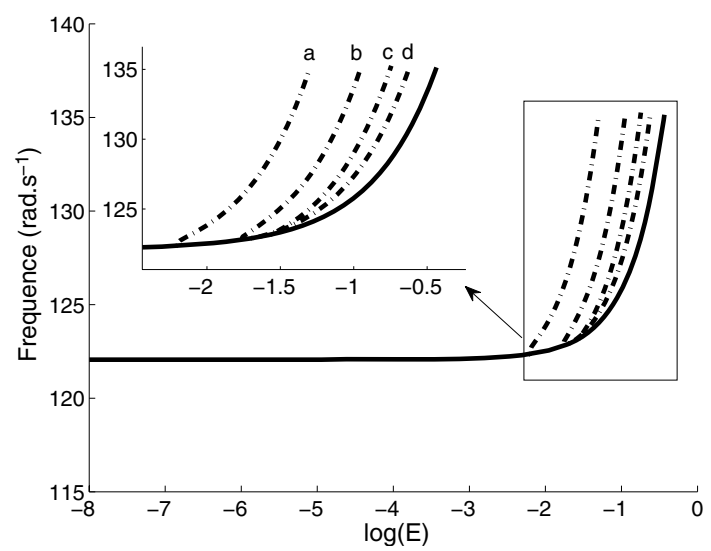

Fig. 7. Backbone curve du mode à 3 diamètres $(-)$ et ses bifurcations localisées $(-\cdot-)(\mathrm{a}: 1$ secteur, $\mathrm{b}: 2$ secteurs, c : 3 secteurs, $d: 4$ secteurs $)$.

évidemment, cette complexité va ressurgir dans le cas du régime forcé. Nous nous proposons d'étudier l'impact des différents types de chargement sur la structure de ces réponses. On s'intéresse donc à la réponse forcée du système à 6 degrés de liberté. Les paramètres numériques utilisés pour le calcul de la réponse forcée sont ceux qui ont été définis à l'équation (8). Dans le but d'obtenir des 


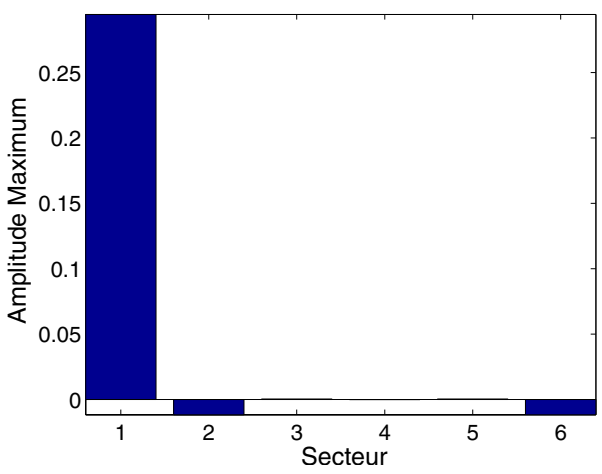

(a) Mode localisé sur 1 secteur

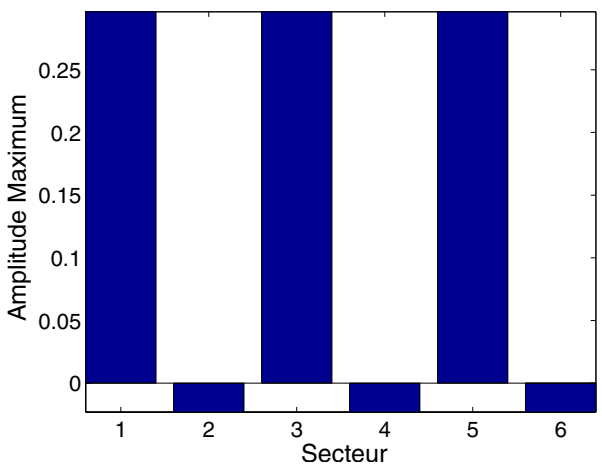

(c) Mode localisé sur 3 secteurs

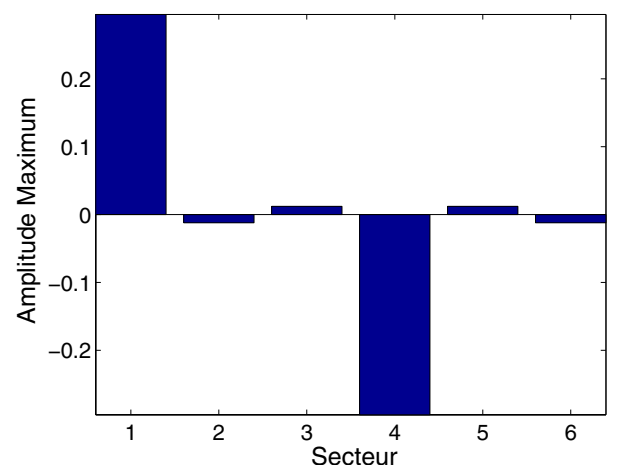

(b) Mode localisé sur 2 secteurs

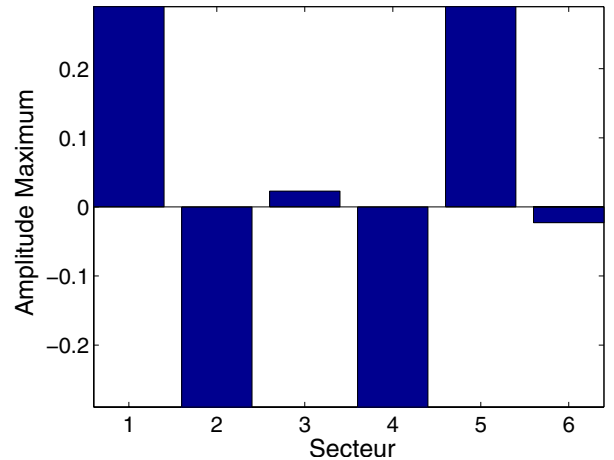

(d) Mode localisé sur 4 secteurs

Fig. 8. Forme propres des bifurcations du mode à 3 diamètres pour une pulsation $\omega=135$ rad.s ${ }^{-1}$.

courbes d'amplitudes finies, on ajoute un terme d'amortissement au système (1) qui devient alors :

$$
\ddot{\boldsymbol{X}}+\delta \dot{\boldsymbol{X}}+\mathbf{K} \boldsymbol{X}+\gamma \boldsymbol{X}^{2}+\beta \boldsymbol{X}^{3}=\boldsymbol{F}(t)
$$

avec $\delta$ une constante définie par $\delta=\omega_{0} / 200$.

On considère que le second membre $\boldsymbol{F}(t)$ se met sous la forme :

$$
\boldsymbol{F}(t)=\boldsymbol{A}_{F} \cos (\Omega t)
$$

où le vecteur $\boldsymbol{A}_{F}$ représente la forme et l'amplitude de la force.

Une étude de la convergence des solutions et de leurs stabilités, montre que nous obtenons de bonnes approximations en ne retenant que 3 harmoniques, les solutions forcées du système (14) seront donc recherchées sous la forme suivante :

$$
\begin{aligned}
\boldsymbol{X}(t)= & \boldsymbol{A}_{0}+\boldsymbol{A}_{1} \cos (\Omega t)+\boldsymbol{B}_{1} \sin (\Omega t) \\
& +\boldsymbol{A}_{2} \cos (2 \Omega t)+\boldsymbol{B}_{2} \sin (2 \Omega t) \\
& +\boldsymbol{A}_{3} \cos (3 \Omega t)+\boldsymbol{B}_{3} \cos (3 \Omega t)
\end{aligned}
$$

Pour l'étude du système forcé, on considérera deux types d'excitation : une excitation sur le premier mode linéaire et une excitation detunée sur le premier mode propre linéaire. Pour ces deux cas, on étudie l'influence du niveau d'excitation sur la réponse du système.

\subsection{Excitation sur le premier mode propre linéaire}

On étudie dans cette section la réponse forcée du système lorsque celui-ci est excité par une force harmonique qui prend la forme du premier mode propre linéaire, c'est-à-dire que l'on considère une force d'excitation du type :

$$
\boldsymbol{F}(t)=\boldsymbol{A}_{F} \cos (\Omega t) \quad \text { avec } \quad \boldsymbol{A}_{F}=A_{F} \boldsymbol{\Phi}_{\mathbf{0}}
$$

où $\boldsymbol{\Phi}_{\mathbf{0}}$ correspond à la forme propre du mode de vibration à zéro diamètre définie à l'équation (11).

Pour de très faibles amplitudes d'excitation $\left(A_{F}=1\right)$, la réponse non-linéaire est analogue à la réponse linéaire (Fig. 9a) et les solutions sont stables pour toutes les fréquences d'excitation.

En augmentant l'amplitude de la force $\left(A_{F}=5\right)$, on fait apparaître une zone d'instabilité entre deux points de retournement (Fig. 9b).

Pour des amplitudes de force élevées $\left(A_{F}=10\right)$, une deuxième zone d'instabilité est générée après le second point de retournement (Fig. 10). Cette deuxième zone instable prend naissance au travers d'une bifurcation de type point d'embranchement. On peut alors calculer une branche bifurquée (Fig. 11) dont l'évolution temporelle et la forme de la solution au pic de la branche bifurquée sont représentées sur la figure 12. Notons que la solution bifurquée possède des zones stables. 


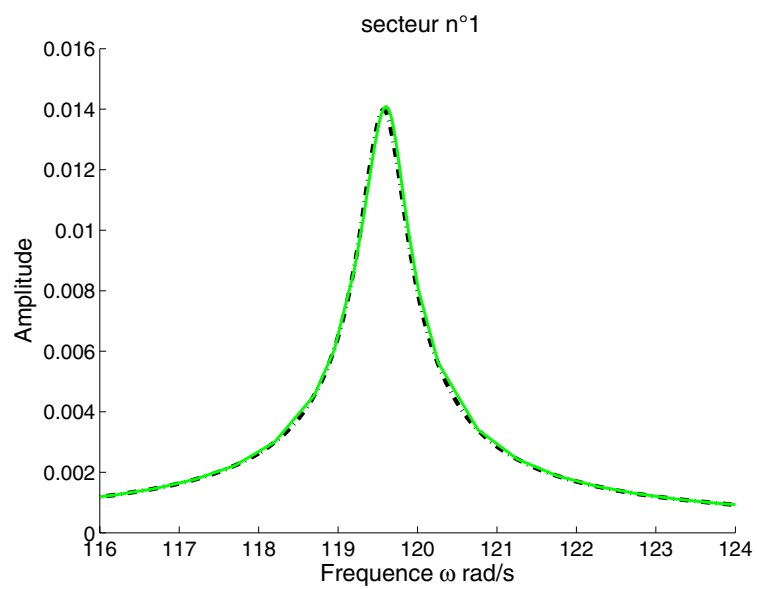

(a) $A_{F}=1$

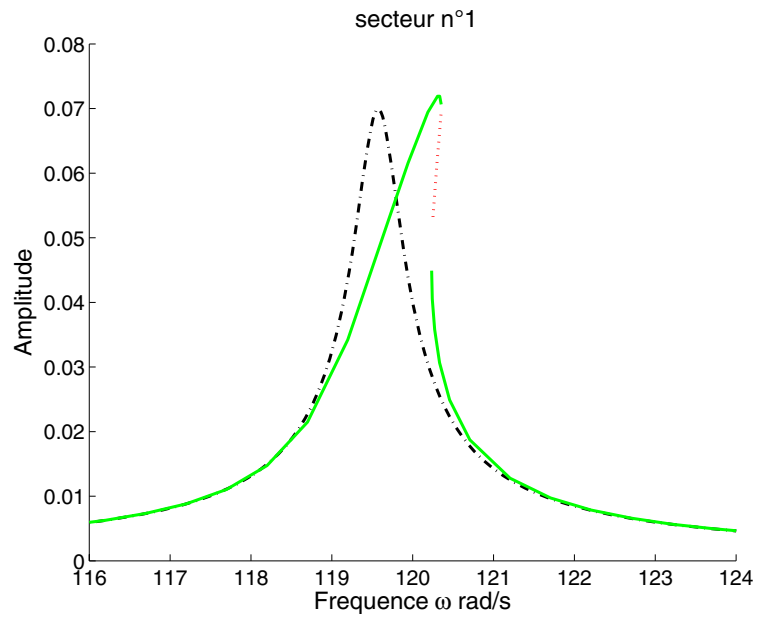

(b) $A_{F}=5$

Fig. 9. Réponse forcée non-linéaire pour une excitation sur le mode à zéro diamètre avec $A_{F}=1$ et $A_{F}=5$ ( - : stable, $\cdots$ : instable, $-\cdot-$ : réponse linéaire).

\subsection{Excitation detunée sur le mode à zéro diamètre}

Il convient de garder à l'esprit que les sollicitations réelles sont souvent imparfaites et correspondent plus à des excitations à diamètre perturbé plutôt qu'à diamètre parfait. Dans cette section, notre étude porte sur le mode de vibration à zéro diamètre, cependant des résultats équivalents pourront être obtenus pour les autres modes de vibration. Nous considérons que seule la première composante de la force est perturbée. Dans ce cas la force d'excitation est donnée par :

$$
\boldsymbol{F}(t)=A_{F}\left(\begin{array}{c}
1+\epsilon_{1} \\
1 \\
1 \\
1 \\
1 \\
1
\end{array}\right) \cos (\Omega t)
$$

où $\epsilon_{1}$ représente le pourcentage de detuning de la force à 0 diamètre. Dans toute la suite on considère que $A_{F}=10$.
On commence les simulations avec un detuning de $1 \%$. En raison des produits scalaires (projections) utilisés dans la HBM, perturber la force d'excitation d'une telle manière revient à ajouter une perturbation à la fonction $\boldsymbol{G}(\boldsymbol{Y}, \omega)$ (Éq. (10)) comme dans le cas d'une recherche de bifurcation par la méthode de perturbation [14]. La perturbation permet alors de détruire les points d'embranchement qui sont structurellement instables. Ainsi les éventuelles bifurcations d'un cas d'excitation à diamètre parfait vont se transformer en des parties de la solution qui seront accessibles par continuation. Cette remarque est vérifiée sur la figure 13, où on observe que la bifurcation du cas $\epsilon_{1}=0$ (Fig. 11) fait désormais partie de la courbe obtenue par continuation.

En augmentant le detuning, les amplitudes des solutions augmentent et, à partir d'un détuning d'environ $40 \%$, il est possible de mettre en évidence une solution sous forme de courbes fermées non raccordées à la solution principale. Ces courbes fermées sont représentées sur la figure 14 pour un detuning de $50 \%$. Cette deuxième famille de solution correspond à un mode de vibration fortement localisé. Ces solutions secondaires se raccordent à la solution principale pour un detuning au voisinage de $70 \%$ (Fig. 15). Etant donné que ces courbes fermées possèdent des zones stables qui correspondent à une amplitude de vibration conséquente (au moins égale à l'amplitude du cas $\epsilon_{1}=0$ ), elles doivent être prises en compte lors du dimensionnement des structures. En effet un changement dans les conditions initiales ou un choc en fonctionnement pourraient permettre d'accrocher de telles solutions et solliciter fortement une des sous-structures.

Pour obtenir les courbes fermées, on choisit un point de départ sur la branche de la FRF localisée pour $\epsilon_{1}=$ $70 \%$, puis on réalise une continuation séquentielle à fréquence fixée en faisant décroitre $\epsilon_{1}$ de 70 à $50 \%$. On applique ensuite l'algorithme de continuation par longueur d'arc sur le point obtenu, on obtient ainsi les solutions secondaires pour le cas $\epsilon=50 \%$. Cette méthode de détection des solutions secondaires est limitée puisqu'elle nécessite le calcul d'une solution pour laquelle les courbes fermées se sont déjà raccordées à la FRF classique dans le but d'obtenir un point d'initialisation pour la continuation décroissante en detuning (pour un detuning de $70 \%$ dans cet exemple). De plus, on ne dispose pas d'informations permettant d'estimer les valeurs de detuning pour lesquelles les courbes fermées vont se raccorder : ces valeurs sont obtenues par essais successifs. Enfin dans le cas où les solutions secondaires ne se racordent jamais à la solution principale cette méthode est inadaptée et inefficace. Une stratégie pour pallier à ces limitations serait de considérer la résolution du système (10) par une méthode d'homotopie [17], ce qui permettrait d'obtenir toutes les solutions pour un cas de chargement.

Dans notre exemple, les solutions sous forme de courbes fermées apparaissent pour un detuning d'environ $40 \%$, cette valeur est relativement élevée. Cependant, l'apparition des courbes fermées est fortement conditionnée par l'amortissement, plus l'amortissement est grand, plus il faut un niveau élevé de détuning pour 
secteur $n^{\circ} 1$
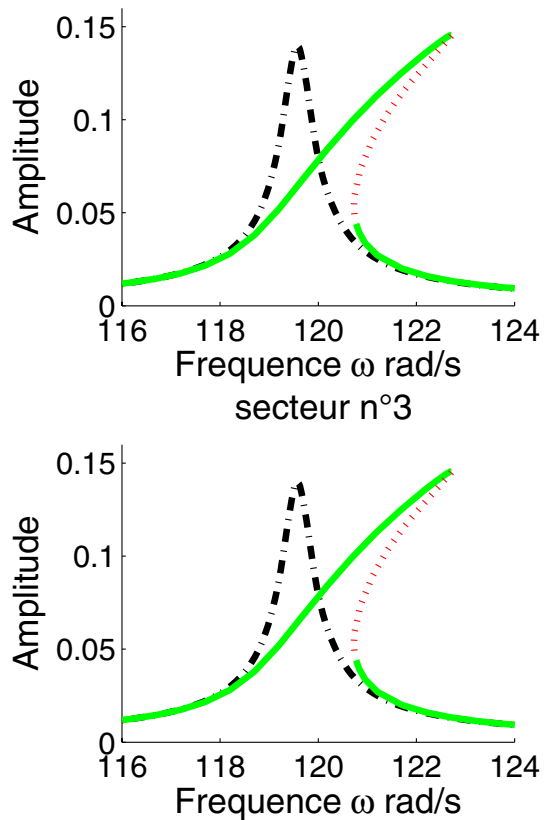

secteur $n^{\circ} 2$
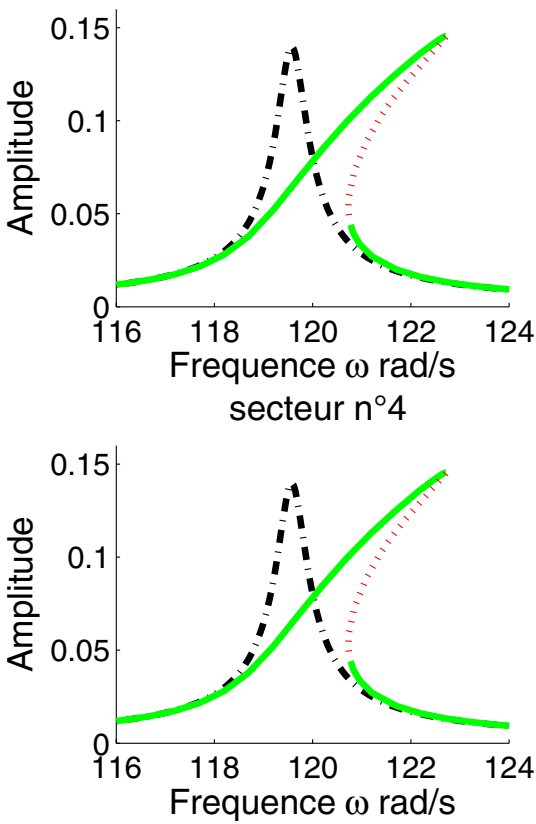

Fig. 10. Réponse forcée non-linéaire pour une excitation sur le mode à zéro diamètre avec $A_{F}=10(-:$ stable, $\cdots:$ instable, $-\cdot-$ : réponse linéaire).

secteur $n^{\circ} 1$
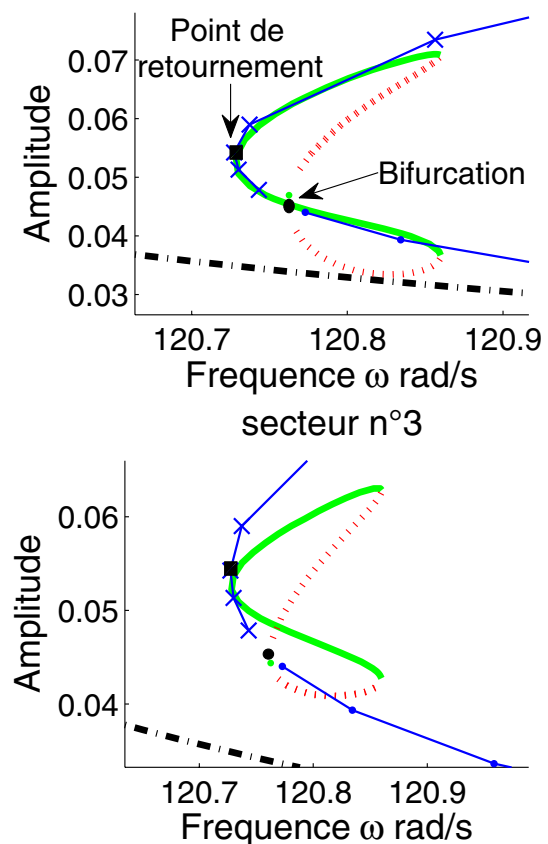

secteur $\mathrm{n}^{\circ} 2$
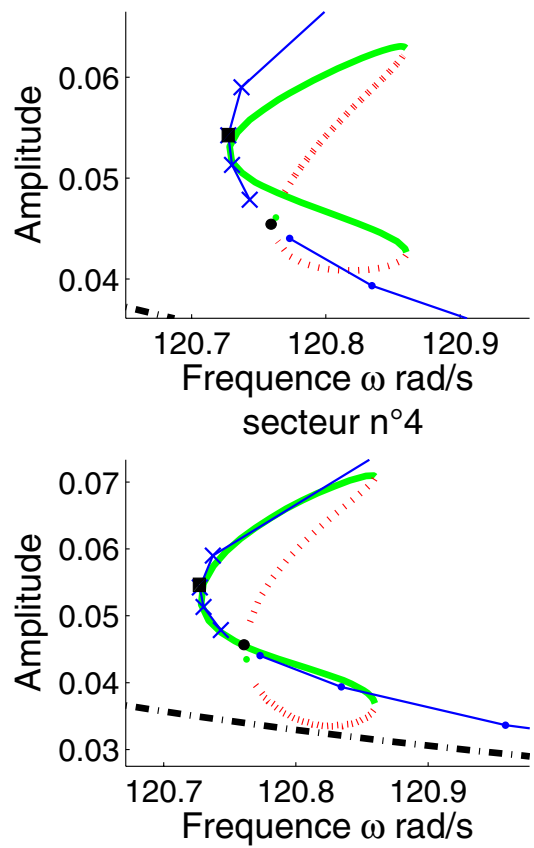

Fig. 11. Branche bifurquée pour une excitation sur le mode à zéro diamètre avec $A_{F}=10(-:$ stable, $\cdots:$ instable, $-\cdot-$ : réponse linéaire).

avoir apparition des courbes fermées et plus leur « temps de vie » est court puisqu'elles vont rapidement se raccorder à la solution non-linéaire classique.

\section{Conclusion}

Dans ces travaux, on a calculé la réponse libre et forcée d'une structure à symétrie cyclique soumise à des non- linéarités géométriques. La mise en équation, discrétisée par la méthode de Ritz, conduit à un système d'équations non-linéaires dont les paramètres ont été ajustés à partir d'une structure réelle. Les modes non-linéaires ainsi que les réponses forcées du système ont été calculés par la méthode de la balance harmonique et la stabilité des solutions par la théorie de Floquet. En plus des modes nonlinéaires issues des modes propres linéaires, on a mis en 


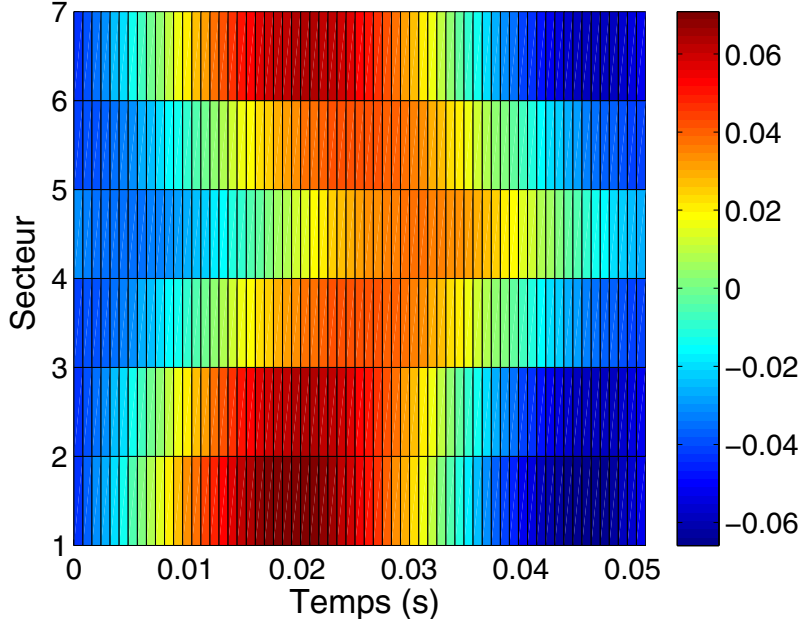

(a) Evolution temporelle

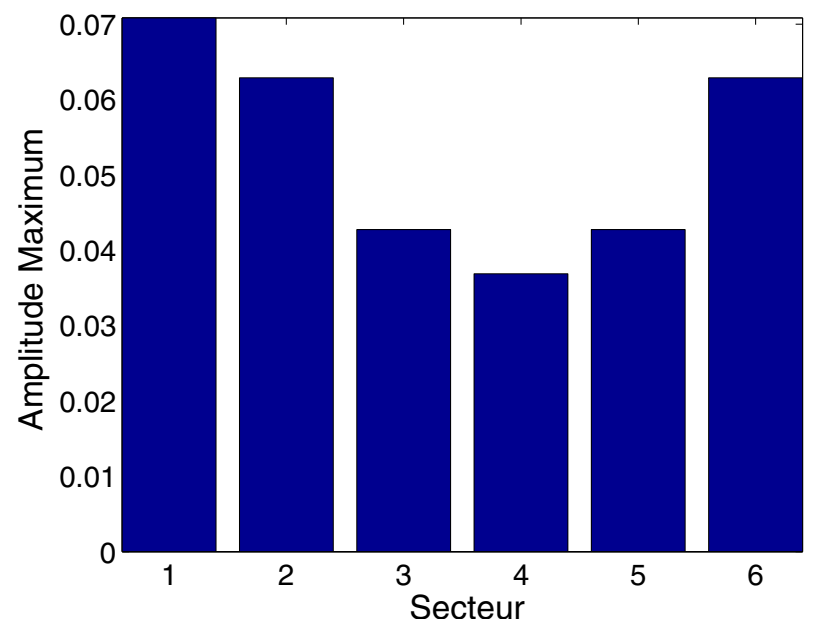

(b) Amplitude maximum

Fig. 12. Évolution temporelle et forme de la solution au pic de la branche bifurquée.
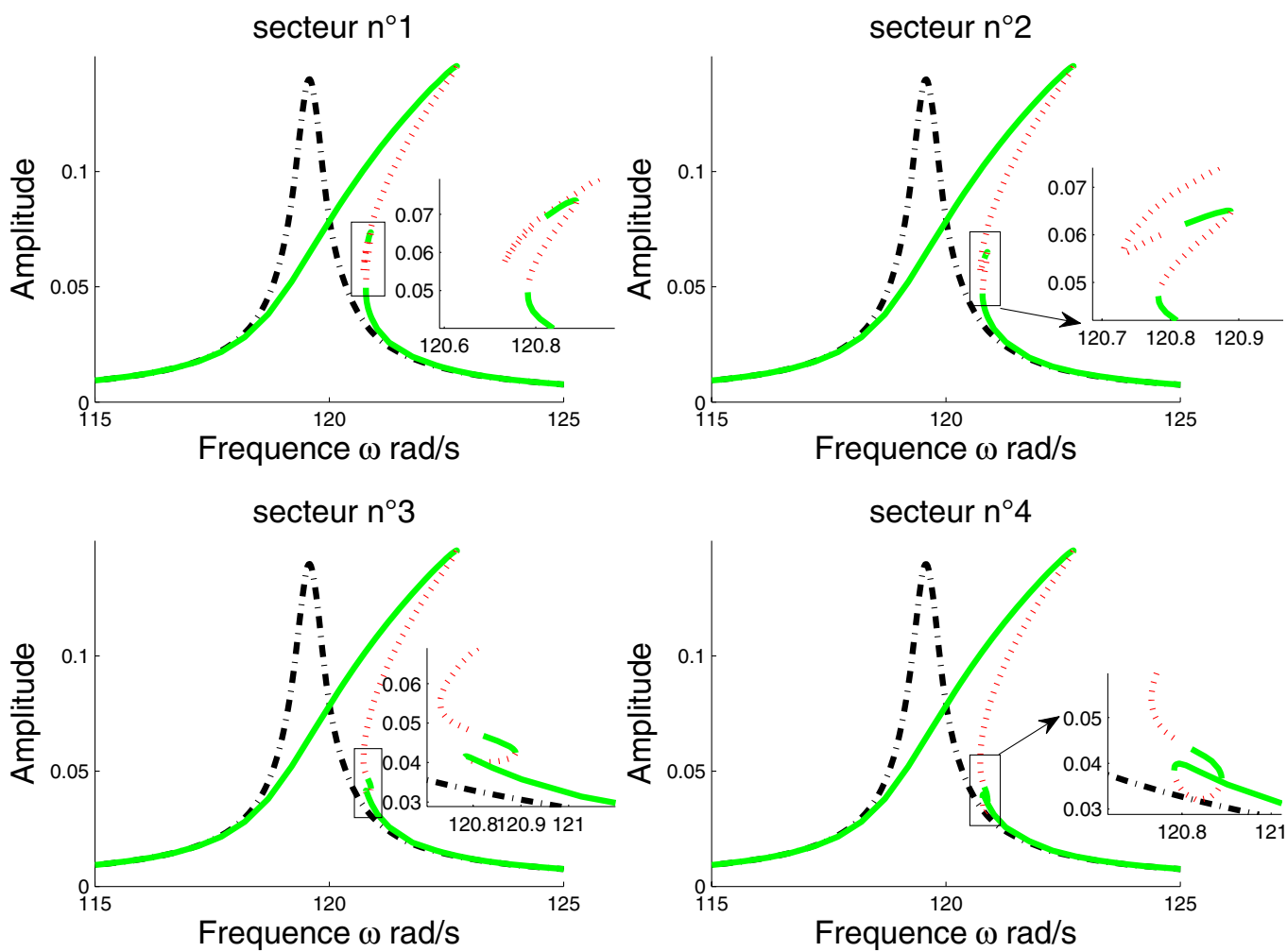

Fig. 13. Réponse forcée non-linéaire pour une excitation sur le mode à zéro diamètre détunée à $1 \%(-:$ stable, $\cdots$ : instable, $-\cdot-$ : réponse linéaire).

évidence d'autres modes de vibration possibles qui sont issus des modes non-linéaires naturels par bifurcation. Certains de ces modes bifurqués correspondent à des modes de vibration plus ou moins localisés. Pour une excitation sur le mode à zéro diamètre, on a mis en évidence des bifurcations de type point d'embranchement qui peuvent également correspondre à des modes de vibration stables. Dans le cas d'une excitation detunée, en plus des réponses classiques, on a mis en évidence un type de solution qui se présente sous la forme de courbes fermées. Ces courbes fermées viennent se coller aux solutions classiques lorsque l'amplitude de la force augmente, engendrant ainsi le phénomène de localisation forcée.

Les simulations sont basées sur un modèle simplifié et les résultats restent donc à un niveau théorique. Cependant, cette étude a permis de mettre en valeur la 

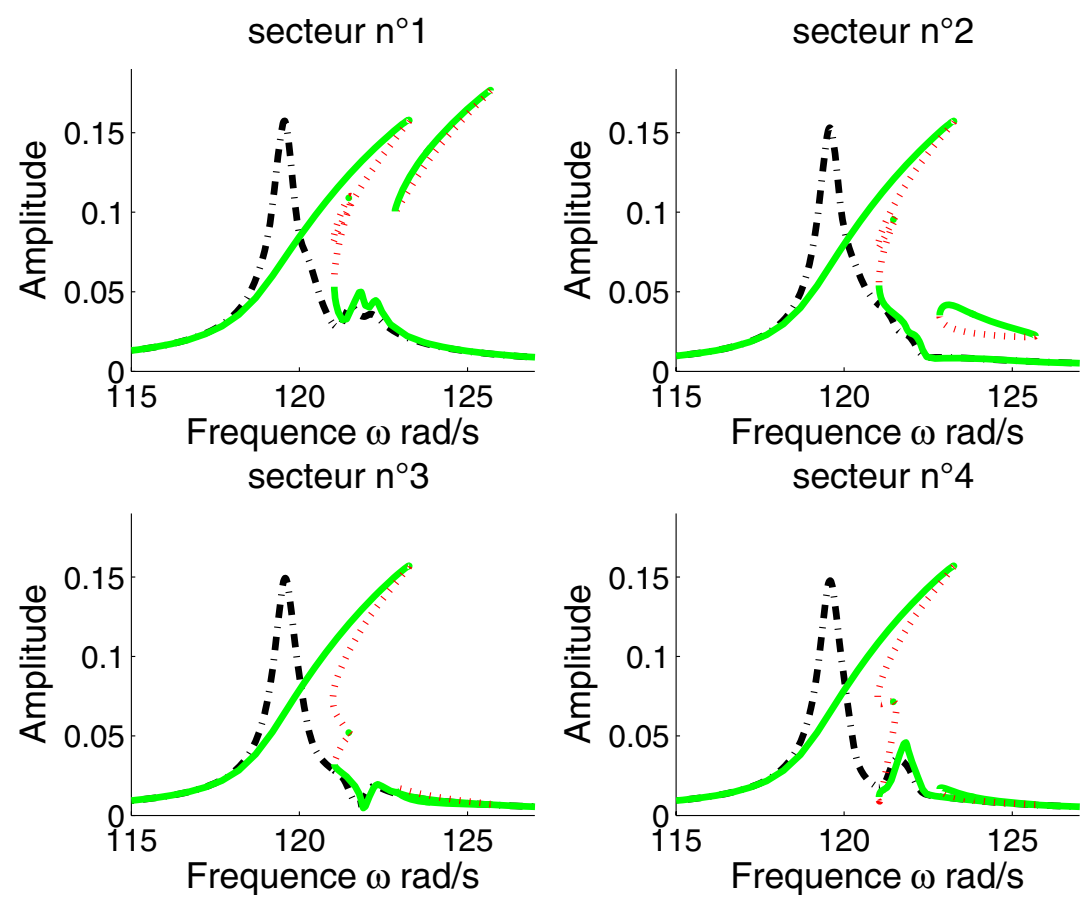

Fig. 14. Réponse forcée non-linéaire pour une excitation sur le mode à zéro diamètre détunée à $40 \%$ (- : stable, $\cdots$ : instable, $-\cdot-$ : réponse linéaire).
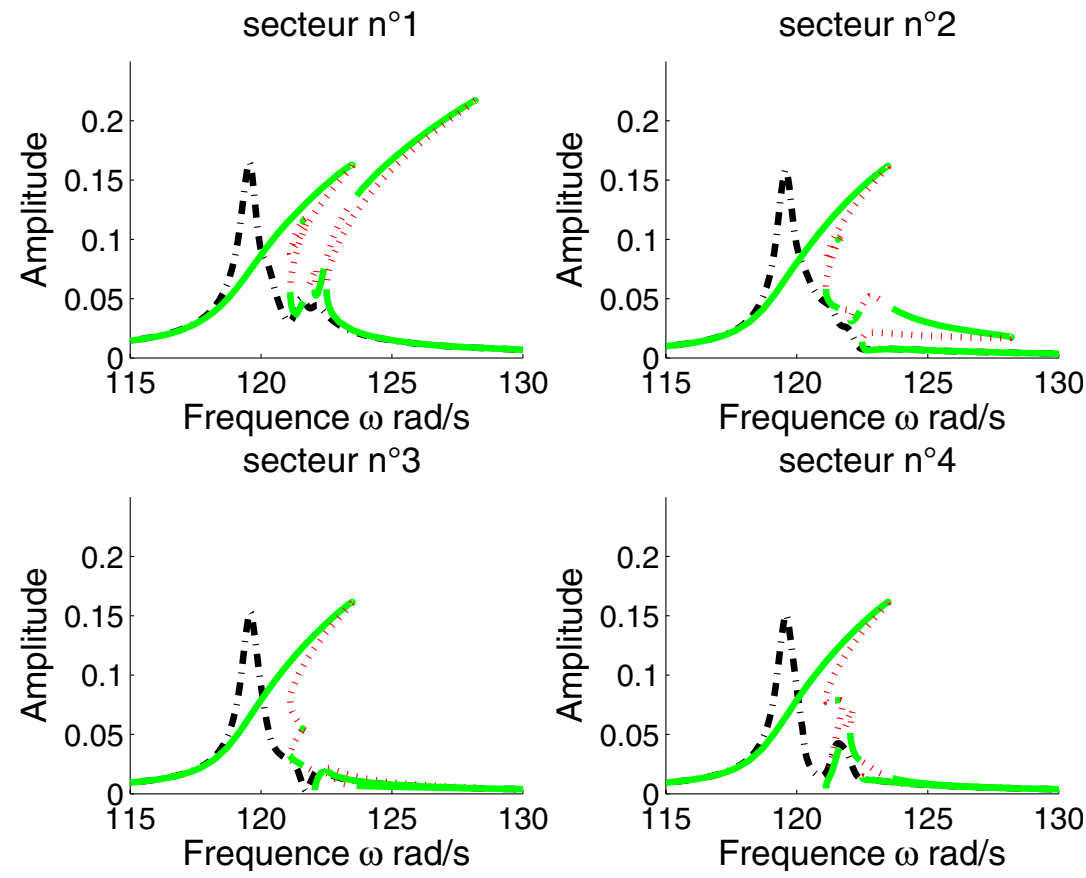

Fig. 15. Réponse forcée non-linéaire pour une excitation sur le mode à zéro diamètre détunée à $70 \%(-:$ stable, $\cdots$ : instable, $-\cdot-$ : réponse linéaire).

complexité des réponses des structures à symétrie cyclique soumise à des non-linéarités géométriques. En particulier, nous avons montré l'existence de poches de solution stables qui peuvent jouer un rôle important lors de la conception de ces systèmes mécaniques. L'estimation de ces solutions obtenues au travers d'une analyse en niveau de chargement des différentes FRF non-linéaires pourrait être améliorée à l'aide d'une recherche systématique de l'ensemble des solutions par exemple par homotopie. Cette stratégie fera l'objet de nos études à venir.

Remerciements. Des remerciements sont adressés à Snecma, pour son support technique et financier. Cette étude prend place dans le cadre du programme de recherche MAIA financé par le CNRS, l'ONERA et le groupe SAFRAN. 


\section{Annexe : Paramètres de l'équation du mouvement}

Dans cet annexe, on donne l'expression des paramètres de l'équation du mouvement pour des secteurs modélisés par des plaques rectangulaires (Éq. (1)). On note $\rho$ la masse volumique, $E$ le module de Young et $\nu$ le ratio de Poisson du matériau. La constante de raideur du couplage linéaire est notée $k$. Les dimensions de la plaque sont notées $L_{x}$ pour la longueur $L_{y}$ pour la largeur et $h$ pour l'épaisseur. Dans ce cas on a :

$$
\begin{aligned}
m & =\frac{\rho h L_{x} L_{y}}{5} \\
a & =\frac{5}{3} \frac{E h^{2}}{\rho L_{x}^{4}\left(1-\nu^{2}\right)} \\
b & =\frac{8 E}{\rho L_{x}^{4}\left(1-\nu^{2}\right)} \\
c & =\frac{1}{256} \frac{k}{m} \\
F^{j} & =\frac{f^{j}(t)}{m}
\end{aligned}
$$

\section{Références}

[1] F. Georgiades, M. Peeters, G. Kerschen, J.C. Golinval, Modal analysis of a nonlinear periodic structure with cyclic symmetry, AIAA J. 47 (2009) 195-216

[2] S. Samaranayake, Subharmonic oscillations in harmonically excited mechanical systems with cyclic symmetry, J. Sound Vib. 206 (1997) 39-60

[3] A.F. Vakakis, Nonlinear normal mode and their application in vibration theory: An overview, Mech. Syst. Signal Process. 11 (1996) 3-22

[4] G. Kerschen, M. Peeters, J.C. Golinval, A.F. Vakakis, Nonlinear normal modes, part I: A useful framework for the structural dynamicist, Mech. Syst. Signal Process. 23 (2009) 170-194
[5] M. Peeters, G. Kerschen, R. Viguié, G. Sérandour, J.C. Golinval, Nonlinear normal modes, part II: toward a practical computation using continuation technique, Mech. Syst. Signal Process. 23 (2009) 195-216

[6] A.F. Vakakis, Normal mode and localisation in nonlinear systems, Wiley-Interscience, 1996

[7] R. Benamar, M.M.K. Bennouna, R.G. White, The effect of large vibration amplitudes on the mode shapes and natural frequencies of thin elastic structures, part II: fully clamped rectangular isotropic plates, J. Sound Vib. 164 (1993) 295-316

[8] M. Amabili, Theory and experiments for large-amplitude vibrations of rectangular plates with geometric imperfections, J. Sound Vib. 291 (2006) 539-565

[9] K.M. Liew, C.M Wang, pb2-Rayleigh-Ritz method for general plate analysis, Eng. Struct. 15 (1993) 55-60

[10] D. Laxalde, F. Thouverez, J.J. Sinou, J.P. Lombard, Qualitative analysis of forced responce of blisks with friction ring dampers, Eur. J. Mech. Solids 36 (2007) 676-687

[11] D. Laxalde, F. Thouverez, Complex nonlinear modal analysis for mechanical systems: application to turbomachinery bladings with friction interfaces, J. Sound Vib. 322 (2009) 1009-1025

[12] R. Lewandowski, Computational formulation for periodic vibration of geometrically nonlinear structures-part 1: theoretical background, Int. J. Solids Struct. 34 (1997) 1925-1947

[13] P. Ribeiro, M. Petyt, Nonlinear vibration of plates by the hierarchical finite element and contination method, Int. J. Mech. Sci. 41 (1999) 437-459

[14] A.H. Nayfey, B. Balanchandran, Applied nonlinear dynamics, Wiley-Interscience, 1995

[15] M. Ribeiro, M. Petyt, Nonlinear free vibration of isotropic plates with internal resonance, Int. J. Non-Linear Mech. 35 (2000) 263-278

[16] R.M. Rosenberg, On nonlinear vibration of systems with many degrees of freedom, Adv. Appl. Mech. (1966) 155242

[17] E. Sarrouy, Analyse globale de systèmes mécaniques nonlinéaires, Application à la dynamique des rotors, Ph.D. thesis, École Centrale Lyon, 2008 